\section{Incidence of Chromophobe Adenoma after Chronic Diisopropylfluorophosphate Poisoning}

IT is widely assumed that changes in brain cholinesterase would have far reaching consequences on brain function. In practice, however, it has been found that, subject to the animal surviving the initial toxic consequences of the cholinesterase reduction, the ensuing effects are not seriously incapacitating.

Using an organophosphate-diisopropylfluorophosphate (DFP) - as the chief irreversible inhibitor of cholinesterases, we have maintained animals on 20-30 per cent of their normal concentration of brain acetylcholinesterase $^{1}$. These animals have be€n used for behavioural testing and for concomitant biochemical study ${ }^{2,3}$. During the experiments, batches of normal and chronic DFP treated rats had to be kept for increasingly longer periods ranging from weeks to $2 \mathrm{yr}$.

Pituitary tumours were found in a batch of rats aged approximately 12-16 months, which had been on a chronic DF'P regimen. Because pituitary tumours had never bcen observed before, closer attention was given to the incidence of this tumour and other tissue abnormalities in rats treated chronically with DFP.

Female Wistar rats were introduced to the laboratory in batches of twenty at 100 days of age. They were caged singly in an air-conditioned colony room at $70^{\circ}$ Fahrenheit with a $12 \mathrm{~h}$ cycle of light and dark. A dose of $1 \mathrm{mg} / \mathrm{kg}$ body weight of DFP was administered at first, followed by booster doses of $0.5 \mathrm{mg} / \mathrm{kg}$ every $72 \mathrm{~h}$. The DFP was made up in arachis oil and was injected intramuscularly (gastrocnemius).

Out of five batches consisting initially of one hundred rats, sixteen confirmed cases of pituitary abnormality were recorded, 12,16 and 18 months after the onset of treatment. This figure does not include those animals which died from causes not established at the time, or which were destroyed because of disease.

The tumours ranged in size from a barely recognizable increase of tissue mass to one with a diameter of $8 \mathrm{~mm}$ and with a wet weight of $99.7 \mathrm{mg}$. The pituitaries were sectioned and stained with haematoxylin and eosin, PAS and Gomori's aldehyde-fuchsin stain. Hyperplasia of the chromophobes was the pathological picture in all but three cases; the remainder showed hypertrophy of the chromophobes. In one specimen cellular pleomorphism and the presence of mitotic figures suggested early malignancy.

Routine post-mortem examinations of all normal and DFP treated rats were conducted in the last 12 months. All pituitaries, whether normal or abnormal in appearance, were sent to the Institute of Medical and Veterinary Science, Adelaide, South Australia, for a pathologist's report. The post-mortem examinations suggested that some of the rats which had been chronically treated with DFP for long periods and which had been recorded in the past as having died from causes unknown may have died because of increased intracranial pressure and brain displacement caused by pituitary overgrowth. All pituitaries, irrospective of appearance, taken from rats which had been chronically treated with DFP for more than approximately 12 months contained hyperplastic chromophobes. The incidence of macroscopic abnormalities approached 100 per cent after chronic treatment with DFP for 21 to 23 months. There are now eleven animals in this latter category. The incidence of nonpituitary tumours was found to be compatible with published observations, indicating an age dependency in their natural occurrence ${ }^{4,5}$.

For experimental purposes not directly associated with this communication, five groups of six female Wistar rats, aged 3 months, were treated with various regimens of DFP. For the first 12 weeks the animals were treated with the chronic regimen already stated. This was followed by a series of injections, spaced $72 \mathrm{~h}$ apart, containing higher concentrations of DFP. The five groups received their final injections in concentrations of either $0.5,0.75,1 \cdot 0$, $\mathrm{I} .5$ or $2.5 \mathrm{mg} / \mathrm{kg}$ body weight. The animals were decapitated and the pituitaries sent, identified only by number, for a pathologist's report. In the groups which received 0.5 and $0.75 \mathrm{mg} / \mathrm{kg}$ body weight of DFP there were no abnormalities. The group receiving $1.0 \mathrm{mg} / \mathrm{kg}$ contained two pituitaries with vacuolated cells; that receiving the $1.5 \mathrm{mg} / \mathrm{kg}$ had no marked abnormalities, and in the group receiving $2.5 \mathrm{mg} / \mathrm{kg}$ all six pituitaries had areas where chromophobes appeared more dense, suggesting hyperplasia.

The literature suggests that the incidence of spontaneous chromophobe adenoma in the rat is $\operatorname{rare}^{4,5}$. Those reported may have been related to the strain of rat used and to the fact that the animals were carrying experimentally induced tumours ${ }^{6,7}$. It is of interest to note in this context that in human carcinoma an associated increase in the size of chromophobe cells has been noted ${ }^{8}$.

There has been one specific and detailed histological study of the anterior pituitary of old female rats which is pertinent to this report ${ }^{9}$. Wolfe et al. examined the pituitaries from twenty-six old breeding females, ranging in age from 543-848 days. Two anterior pituitaries were "enlarged, congested and soft". Both were thought to be adenomata of the pituitary. In fifteen rats, 58 per cent of the population, "small areas often consisting of only a few cells" were found which were histologically different from the rest. The difference, in most cases, consisted of "hypertrophied chromophobes". The authors suggest that in their strain of aged breeding females "there is a tendency for atypical areas of hypertrophied cells to appear". In contrast the pituitaries reported here usually showed chromophobe hyperplasia; there was a remarkably high incidence of this abnormality in the animals which received DFP as compared with only one tumour in a control group of equivalent age and sex.

The data obtained by treating some animals with a standard DFP regimen, and treating others with higher concentrations of DFP, suggest that chromophobe abnormalities may be induced in younger females by this treatment. It is possible that DFP has a specific oncogenic effect on the female rat pituitary, or perhaps uncovers or accelerates a change latent in that gland.

This work was supported by the Australian Research Grants Committee. I thank the members of the Institute of Medical and Veterinary Science, Adelaide, South Australia for their assistance.

Peter H. Glow

Department of Psychology,

University of Adelaide,

South Australia.

Received October 25; revised December 18, 1968.

${ }^{1}$ Glow, P. H., Rose, S., and Richardson, A., Austral. J. Biol. Med. Sci., 44, 73 (1966).

${ }^{2}$ Glow, P. H., and Richardson, A., Nature, 214, 629 (1967).

${ }^{3}$ Glow, P. H., Richardson, A., and Rose, S., J. Comp. Physiol. Psychol., 63, 155 (1967).

Bullock, F. D., and Curtis, M. R., Amer. J. Cancer, 14, 1 (1930).

${ }^{5}$ Ratcliffe, N., Amer. J. Path., 16, 237 (1940).

6 Guyer, M. F., and Claus, P. E., Anat. Res., 52, 225 (1932).

Heiman, J., Amer. J. Cancer, 33, 423 (1938).

Karlefors, J., $Z$. Krebsforsch.,17, 195 (1920).

${ }^{\circ}$ Wolfe, J. M., Bryan, W. R., and Wright, A. W., Proc. Soc. Biol. and Med., $38,80(1938)$.

\section{Neurological IIIness after Inoculation of Tissue from Tumour Bearing Animals}

Certain transmissible infectious agents may remain in tissues for prolonged periods before leading to pathological processes and disease ("slow" infections) ${ }^{1}$. Riley 